Bull. Mater. Sci., Vol. 3, Number 2, July 1981, pp. 193-199. (c) Printed in India.

\title{
Correlation between the magnetic properties and the chemical shifts in $x$-ray absorption spectra of some transition metal compounds
}

\author{
CHINTAMANI MANDE and $M$ Y APTE \\ Department of Physics, Nagpur University, Nagpur 440 010, India
}

MS received 20 November 1980

\begin{abstract}
Studies on the chemical shifts of the $K \mathrm{x}$-ray absorption discontinuities of some transition metal compounds recently conducted in our laboratory have revealed that the $\Delta E$ vs $q$ curves do not pass through the origin. For such systems one obtains an intercept on the $\Delta E$ axis, which seems to depend upon $n_{d}$, the number of unpaired $d$ electrons. As is well-known the magnetic properties of the compounds of transition metal elements are governed by the $d$ electrons. It is shown that the magnetic properties of the transition metal compounds may be correlated with $\mathrm{x}$-ray absorption spectra.
\end{abstract}

Keywords. X-ray absorption spectra; chemical shifts; magnetic properties.

\section{Introduction}

It is well-known that the chemical shifts $(\Delta E)$ of an x-ray absorption discontınuity are governed by the effective charge $(q)$ on the absorbing atom (Sapre and Mande 1972, 1973; Ghatikar et al 1977). For the non transition metal compounds it has been shown that the $\Delta E$ vs $q$ curves pass through the origin. However, the results for the compounds of transition metals indicate that the $\Delta E, q$ curves do not pass through the origin, but give rise to some finite intercept $c$ on the $\Delta E$ axis. The magnitudes of such $c$ values appear to be changing with the number $\left(n_{d}\right)$ of the $d$ electrons in the transition metal compounds. As this number $n_{d}$ also decides the magnetic properties to a considerable extent, we considered it worthwhile to see if there exists a correlation between magnetic susceptibility $(\mu)$ and the intercept $c$.

\section{Experimental}

A Machlett X-ray tube with copper target was used as the source of white radiation. A Cauchois type bent crystal $x$-ray spectrograph of diameter $0.40 \mathrm{~m}$, equipped with a well-tested mica crystal oriented to reflect from the (100) planes, was used to photograph the spectra. The wavelength of the absorption discontinuities was measured on their inflexion points on microphotometer records of magnification $\times 100$ using the spectroline scanner (Applied Research Laboratories, USA). A large number of measurements were made on microphotometer traces at different heights of 
the spectrum and the standard error in the measurements was of the order of $+0.5 \mathrm{eV}$.

Pure manganese metal and the different compounds used in this work were of AR grade. Other details of the experimental technique can be found in Ballal and Mande (1977) and Apte (1980).

\section{Results}

Our results on the $K$ absorption discontinuity in pure manganese and its compounds are given in table 1. The wavelength value of the $K$ absorption discontinuity of pure manganese metal is in fairly good agreement with that reported by Cauchois and Senemaud (1978). It is seen that the absorption edges in all the compounds studied in this investigation are shifted to the higher energy side with respect to that in the pure element indicating that the manganese ion behaves as a cation (Sapre and Mande 1972) in all the compounds.

The effective charges on the manganese ion in the compounds are calculated by using Suchet's formula (Suchet 1961)

$$
q=n\left[1-0.01185\left(\frac{Z}{r^{\prime}}+\frac{Z^{\prime}}{r}\right)\right],
$$

where $n$ is the valency, $Z$ is the total number of electrons residing on the cation and $r$ is its ionic radius. The primed symbols refer to the anion in the compound. The

Table 1. Chemical shift of manganese $K$ absorption discontinuity and the effective charge on the manganese ion in the compounds.

\begin{tabular}{|c|c|c|c|}
\hline No & Absorber & $\begin{array}{c}\text { Chemical } \\
\text { Shift, } \Delta E \\
\text { (eV) }\end{array}$ & $\begin{array}{c}\text { Effective } \\
\text { charge } q \\
\text { (electrons/atom) }\end{array}$ \\
\hline 1 & $\mathrm{MnO}$ & 5.5 & 1.31 \\
\hline 2 & $\alpha-M n S$ & 5.0 & 1.17 \\
\hline 3 & $\mathrm{Mn}_{2} \mathrm{O}_{3}$ & 9.9 & 1.91 \\
\hline 4 & $\mathrm{MnO}_{2}$ & 14.0 & 2.48 \\
\hline 5 & $\mathrm{Mn}_{3} \mathrm{O}_{4}$ & 8.2 & 1.70 \\
\hline 6 & $\mathrm{LaMnO}_{3}$ & 11.1 & 1.91 \\
\hline 7 & $\mathrm{MnSO}_{4} \cdot \mathrm{H}_{2} \mathrm{O}$ & 6.2 & $1.38(a)$ \\
\hline 8 & $\mathrm{MnC}_{2} \mathrm{O}_{4} \cdot 2 \mathrm{H}_{2} \mathrm{O}$ & 5.2 & $1.18(a)$ \\
\hline 9 & $\mathrm{MnCl}_{2} \cdot 4 \mathrm{H}_{2} \mathrm{O}$ & 4.9 & $1.14(a)$ \\
\hline 10 & $\mathrm{MnSe}$ & $2.5(b)$ & $0.66(b)$ \\
\hline 11 & $\mathrm{MnTe}$ & $0.90(b)$ & $0.15(b)$ \\
\hline 12 & $\mathrm{MnBr}_{2}$ & $2.55(b)$ & $0.63(b)$ \\
\hline 13 & MnAs & $3.0(b)$ & $0.70(b)$ \\
\hline 14 & $\mathrm{MnP}$ & $5.3(b)$ & $1.20(b)$ \\
\hline 15 & $\mathrm{MnN}$ & $10.3(b)$ & $2.01(b)$ \\
\hline 16 & $\mathrm{CuMnCoO}_{4}$ & $13.8(b)$ & $2.41(b)$ \\
\hline 17 & $\mathrm{~K}_{2} \mathrm{MnF}_{6}$ & $14.0(b)$ & $2.40(b)$ \\
\hline
\end{tabular}

(a) Value calculated from $\Delta E, q$ plot.

(b) Value from Sarode et al (1979) 
effective charges $(q)$ on manganese ions have been calculated directly by using equation (1) in the case of $\mathrm{MnO}, \alpha-\mathrm{MnS}, \mathrm{Mn}_{2} \mathrm{O}_{3}$ and $\mathrm{MnO}_{2}$ and are given in the column 4 of table 1. In the case of $\mathrm{Mn}_{3} \mathrm{O}_{4}$ and $\mathrm{LaMnO}_{3}$ the effective charges on the manganese ions are determined by using some modifications in Suchet's method of charge calculation as stated below.

The molecule $\mathrm{Mn}_{3} \mathrm{O}_{4}$ contains one $\mathrm{Mn}^{2+}$ and one $\mathrm{Mn}^{3+}$ ions. It can be supposed to be a combination of $\mathrm{MnO}$ and $\mathrm{Mn}_{2} \mathrm{O}_{3}$. The charges on the $\mathrm{Mn}^{2+}$ and $\mathrm{Mn}^{3+}$ ions in these compounds are 1.31 and 1.91 electrons/atom respectively, giving the weighted average charge 1.71 electrons/atom on each manganese atom in $\mathrm{Mn}_{3} \mathrm{O}_{4}$.

We consider that in a molecule of $\mathrm{Mn}_{2} \mathrm{O}_{3}$ one atom of manganese is replaced by an atom of lanthanum (57). Each atom of manganese and lanthanum may give three electrons to the three oxygen atoms in the molecule. Thus $Z_{\mathrm{Mn}}=22$ (and $Z_{\mathrm{La}}=54$ ). Each oxygen atom receives two electrons, thereby making $Z_{0}^{\prime}=10$. Taking the ionic radius (Dickinson 1979) for $\mathrm{Mn}^{3+}$ to be $0.66 \mathrm{~A}$ and that for $0^{2-}$ to be $1.40 \mathrm{~A}$ the effective charge $(q)$ on the manganese atom in $\mathrm{LaMnO}_{3}$ comes out to be 1.91 electrons/atom.

Another way of evaluating $q$ in $\mathrm{LaMnO}_{3}$ is to consider separately two compounds $\mathrm{Mn}_{2} \mathrm{O}_{3}$ and $\mathrm{La}_{2} \mathrm{O}_{3}$, in which both the manganese atom and the lanthanum atom are trivalent. The combination of these two compounds will yield the molecular formula $\mathrm{La}_{2} \mathrm{Mn}_{2} \mathrm{O}_{6}$, and which may be considered as $\left(\mathrm{LaMnO}_{3}\right)_{2}$. For $\mathrm{Mn}_{2} \mathrm{O}_{3}$ we obtain $q_{\mathrm{Mn}}=1.91$ and $q_{0}=-1.27$ electrons/atom by using Suchet's formula. Similarly for $\mathrm{La}_{2} \mathrm{O}_{3}$ we get $q_{\mathrm{La}}=+1.32$ and $q_{0}=-0.88$ electrons/atom. When the two individual molecules combine to produce two molecules of $\mathrm{LaMnO}_{3}$, the oxygen atoms in the newly formed $\mathrm{LaMnO}_{3}$ are the same, and hence would have the same magnitude of charge. Let us suppose that each oxygen atom has now an average charge of -1.07 electrons/atom. The charges on manganese and lanthanum atoms would be still the same, i.e. +1.91 and +1.32 electrons/atom thereby giving the total charge neutrality to the $\mathrm{LaMnO}_{3}$ molecule. Kondawar and Mande (1976) have similarly calculated the charge on the cobalt atom in the compound CoAsS by considering the charge on cobalt as an average of its charge in the two compounds $\mathrm{CoAs}_{2}$ and $\mathrm{CoS}_{2}$. In the present case we have estimated the charge on the oxygen (anion) by this method of average.

The $q$ values thus calculated for the compounds $\mathrm{Mn}_{3} \mathrm{O}_{4}$ and $\mathrm{LaMnO}_{3}$ are also included in table 1 .

\section{Discussion}

In order to see the correlation between $\Delta E$ and $q$ we plot in figure 1 , from the values given in table 1, a graph between the two quantities. We can roughly draw a single curve going through most of the points as shown in figure 1 . This curve resembles that of Sapre and Mande $(1972,1973)$ for the positive shifts in arsenic and gallium compounds. Suchet (1975) has proposed from the results of Sapre and Mande that the $\Delta E, q$ curve is represented by the equation

$$
\Delta E=a q+b q^{3}
$$

This equation suggests that the $\Delta E, q$ curve should pass through the origin, as in the case of the curve given by Sapre and Mande. However, we have observed that in 


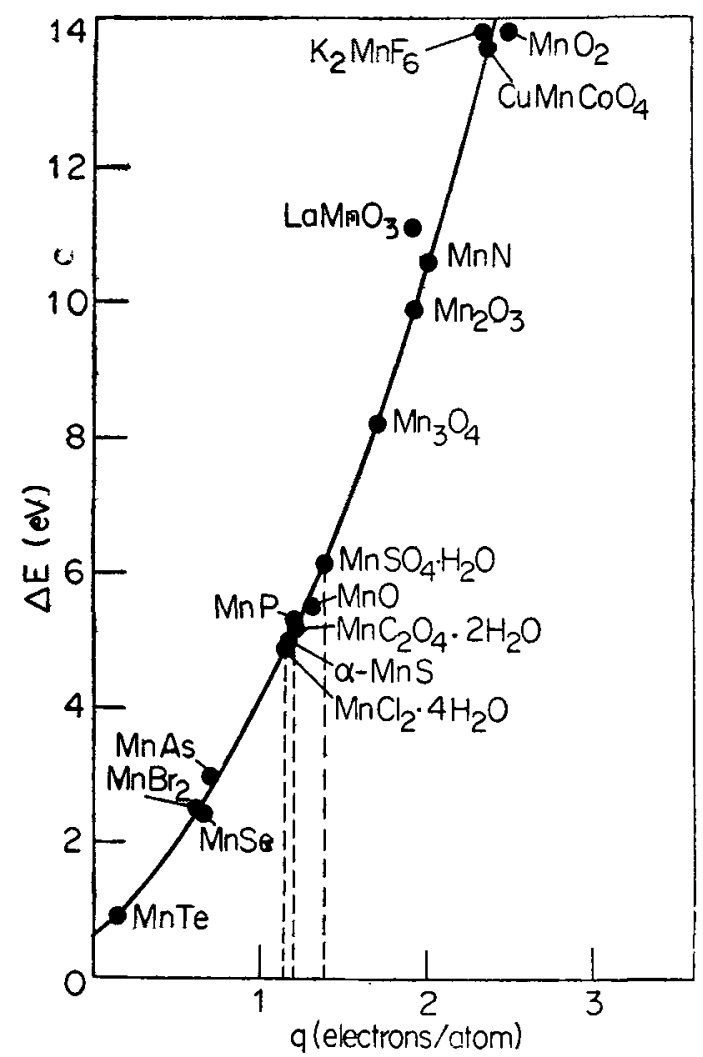

Figure 1. $\Delta E$ vs $q$ plot for manganese compounds

manganese compounds the $\Delta E, q$ curve, although generally similar to that given by Sapre and Mande, does not pass through the origin. It has been reported (Kondawar 1975, Kondawar and Mande 1976) that the $\Delta E, q$ curves in the case of some compounds of cobalt also do not pass through the origin. Mande and Suchet (1978) have critically analysed the results of Kondawar (1975) and Kondawar and Mande (1976) and have suggested that the $\Delta E, q$ curve may be represented in the case of transition metal compounds by the equation

$$
\Delta E=a q+b q^{3}+c
$$

where $c$ is a constant that depends upon the number $\left(n_{d}\right)$ of $d$ electrons in the compounds. Equation (3) suggests that there should be separate $\Delta E, q$ curves for the compounds with different values of $n_{d}$.

In figure 2 we have plotted separate curves for different values of $n_{d}$. We obtain $c=0.6 \mathrm{eV}$ in case of $d^{5}$ divalent manganese compounds $\mathrm{MnO}, \alpha-\mathrm{MnS}, \mathrm{MnSe}, \mathrm{MnTe}$, while the separate curve for trivalent manganese $\left(d^{4}\right)$ compounds $\mathrm{Mn}_{2} \mathrm{O}_{3}, \mathrm{LaMnO}_{3}$, MnAs, MnP and MnN gives $c=0.8 \mathrm{eV}$. Though our data are very scanty we can think of a similar separate curve being drawn for the tetravalent manganese compounds for which $n_{d}=3$. This curve gives a higher $c$ value (about $1.4 \mathrm{eV}$ ) for the 
tetravalent compounds. Kondawar and Mande have studied some cobalt compounds and obtained $c$ values of 1.68 and $2.5 \mathrm{eV}$ for $d^{7}$ and $d^{9}$ compounds respectively. The results of the earlier workers indicate $c=0$ for $n_{d}=0$ and 10 , since the $\Delta E, q$ curves pass very well through the origin. The $c$ values for the different values of $n_{d}$ known so far are given in table 2 .

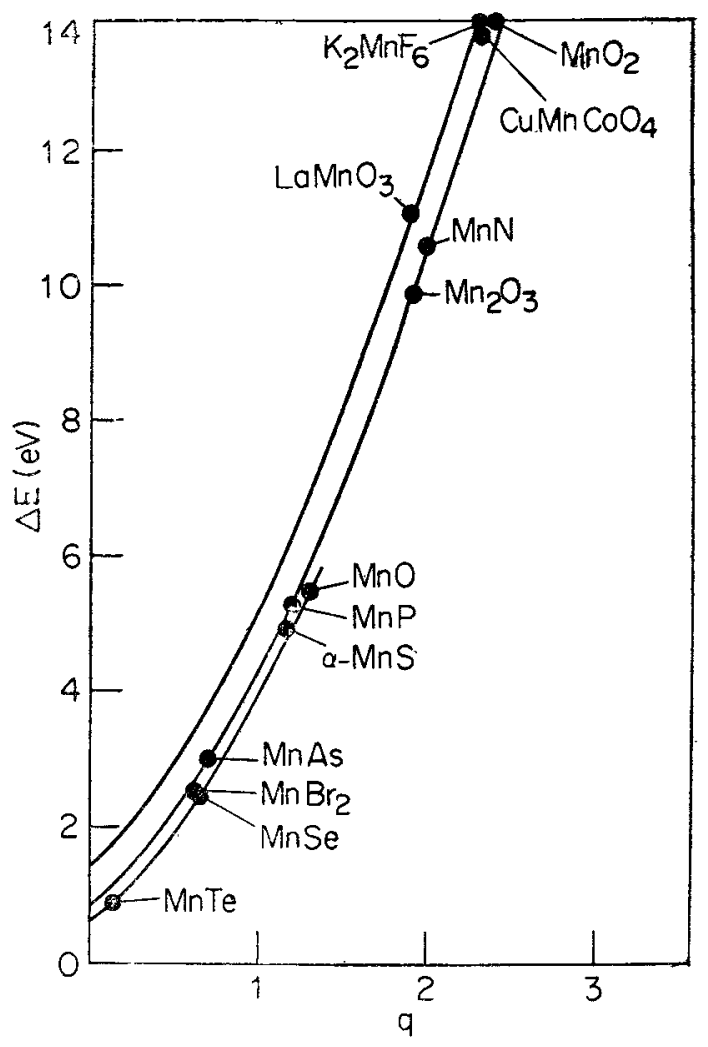

Figure 2. $\Delta E$ vs $q$ plots for manganese compounds for different $n_{d}$ values.

Table 2. Values of $c$ and $\mu$ for different compounds of manganese.

\begin{tabular}{llccc}
\hline No. & Compounds & $n_{d}$ & $c(\mathrm{eV})$ & $\mu$ (B.M.) \\
\hline 1. & $\mathrm{MnO}, \mathrm{MnS}, \mathrm{MnSe}, \mathrm{MnTe}$ & 5 & 0.6 & 5.92 \\
2. & $\begin{array}{l}\mathrm{Mn}_{2} \mathrm{O}_{3}, \mathrm{MnAs}, \mathrm{MnN} \\
\mathrm{MnP}, \mathrm{LaMnO}_{3}\end{array}$ & 4 & 0.8 & 4.90 \\
3. & $\begin{array}{l}\mathrm{MnO}_{2}, \mathrm{~K}_{2} \mathrm{MnF}_{6} \\
\mathrm{CuMnCoO}_{4}\end{array}$ & 3 & 1.4 & 3.88 \\
\hline
\end{tabular}

As suggested by Suchet, (Private Communication) we have plotted in figure 3 , the values of $c$ and $n_{d}$. It will be seen that the $c$ value is minimum for $n_{d}=5$ and that 
it rises on both sides from this point. For $n_{d}=9, c$ has the maximum value. It has zero value for $n_{d}=10$, as seen in the curve for monovalent copper compounds. Thus we actually get the curve for the points for which $n_{d}$ varies between 3 to 10 . Though we do not have any points for $n_{d}<3$, we can draw by extrapolation, a symmetrical curve for that part (as shown by the dotted line) intuitively.

In discussing the chemical shifts in $\mathrm{x}$-ray absorption spectra it has been customary to consider only the change in the position of the outer unoccupied energy level, the inner lcvel being supposed to be unaffected by chemical combination. However, it is well-known that all the levels of the atom are affected by chemical combination, although the effect on the inner levels may be relatively much smaller. It is felt that the $c$ values in our study ace relatcd with the small shifts of the inner $K$ level, as discussed in Apte and Mande (1981).

It is well-known that the magnetic susceptibility $(\mu)$ of a transition-metal compound depends (Pauling 1975) upon the value of $n_{d}$. This suggests that the intercept $c$ may be related to $\mu$, and hence may even be used to ascertain the magnetic properties of the compound. We have shown in the last column of table 2 the calculated values of $\mu$ for the compounds with different values of $n_{d}$. In figure 4 we plot the experi-

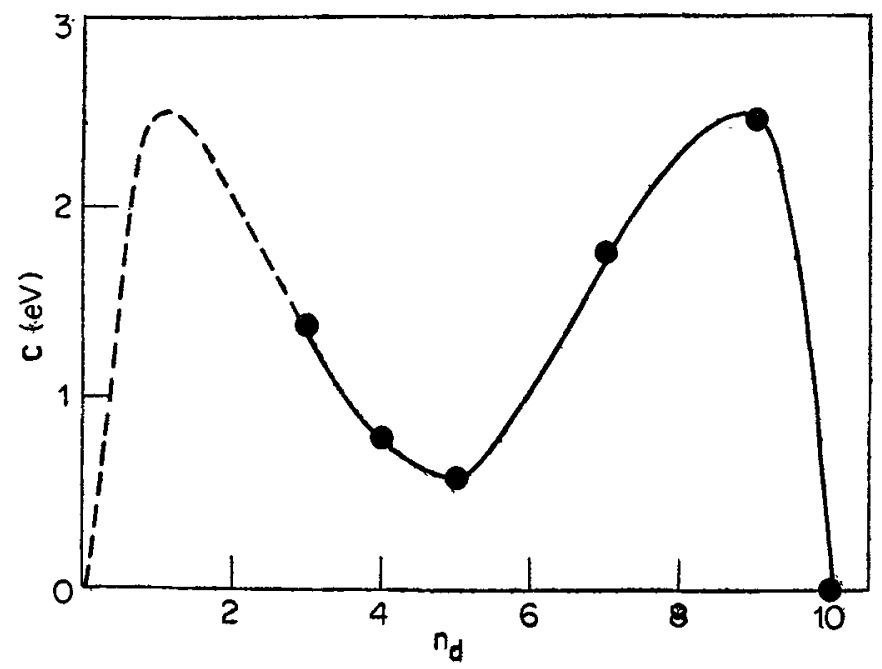

Figure 3. Plot between $n_{d}$ and $c$.

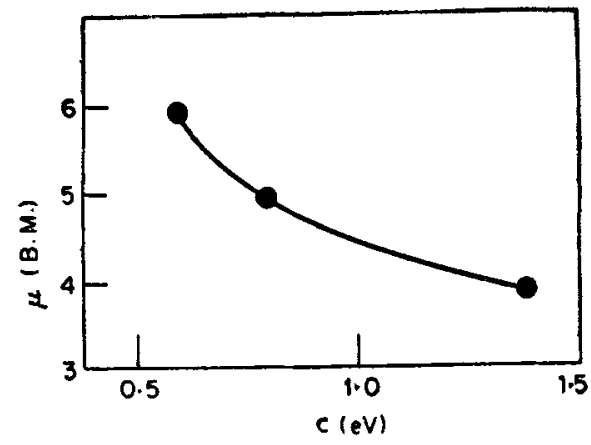

Figure 4. Plot between $c$ and $\mu$. 
mentally determined values of 'c against the values of $\mu$ and we get the minimum $c$ value of $6.6 \mathrm{eV}$ for our manganese compounds for $n_{d}=5$. The change in $n_{d}$ on either side of 5 would decrease the magnetic moment, and hence the point for $c=0.6 \mathrm{eV}$ is a maximum of the curve. The magnetic moment $\mu$ would be zero for the compounds with $n_{d}=0$ or 10 , and for such compounds the $\Delta E, q$ curve may pass through the origin giving $c=0$.

We have for the present only three points in our $\mu v_{s} c$ curve. It will be worthwhile to extend this work to other systems to establish the exact nature of this curve. It may then be possible to know value of $\mu$ for the system in which the magnetic data are not available with the help of figure 4 and this $\mu$ value could give the number of unpaired $d$ electrons in the ion and the type of bonding in the compound.

\section{References}

Apte M Y 1980 Chemical effects in $x$-ray absorption spectra Ph.D. Thesis Nagpur University Apte M Y and Mande C 1981 J. Phys. C (Communicated)

Ballal M M and Mande C 1977 J. Phys. Chem. Solids 38845

Cauchois $Y$ and Senemaud C 1978 International tables of selected constants, wavelength of $x$-ray emission lines and absorption edges (Oxford: Pergamon Press)

Dickinson S K Jr. 1970 Ionic, covalent and metallic radii of the chemical elements Air Force Cambridge Research Laboratories, L. G. Hanson Field, Bedford, Massachusetts USA Physical Science Research Paper No 439

Ghatikar M N, Padalia B D and Nayak R M 1977 J. Phys. C 104173

Kondawar V K 1975 Study of matter by $x$-rays Ph.D. Thesis Nagpur University

Kondawar V K and Mande C 1976 J. Phys. C9 1351

Mande C and Suchet J P 1978 Solid State Chemistry European Meeting, France Strasbourg, III B22

Pauling $\mathrm{L} 1975$ The nature of chemical bond and the structure of molecules and crystals (New Delhi: Oxford and IBH Publishing Co.)

Sapre V B and Mande C 1972 J. Phys. C5 793

Sapre V B and Mande C 1973 J. Phys. Chem. Solids 341351

Sarode P R, Ramasesha S, Madhusudan W H and Rao C N R 1979 J. Phys. C12 2439

Suchet J P 1961 J. Phys. Chem. Solids 21156

Suchet J P 1975 C. R. Acad. Sci. Paris 281-87 